\title{
Suicidal and self-injurious behavior among patients with alcohol and drug abuse
}

\author{
This article was published in the following Dove Press journal: \\ Substance Abuse and Rehabilitation \\ 17 July 2012 \\ Number of times this article has been viewed
}

\author{
Abdullah Mohammed \\ Al-Sharqi' \\ Khaled Saad Sherra² \\ Abdulhameed Abdullah \\ Al-Habeeb ${ }^{3}$ \\ Naseem Akhtar Qureshi ${ }^{3,4}$ \\ 'Private Clinic, Riyadh, Saudi Arabia; \\ ${ }^{2}$ Psychiatric Department, Faculty \\ of Medicine, Mansoura University, \\ Egypt; ${ }^{3}$ General Administration for \\ Mental Health and Social Services, \\ ${ }^{4}$ General Directorate of Research and \\ Studies, Ministry of Health, Riyadh, \\ Saudi Arabia
}

Background: Self-injurious behavior, a major public health problem globally, is linked with alcohol and drug abuse. This cross-sectional study aimed to identify the prevalence and correlates of self-harming behavior in patients with alcohol or drug abuse problems.

Methods: This was a one-year study that recruited a convenience sample of 736 outpatients and inpatients identified with alcohol or drug abuse, and was conducted at Al-Amal mental health hospitals in three major cities. All consecutively selected patients were interviewed on five working days for data collection on a semistructured sociodemographic form using the Columbia Suicide Severity Rating Scale Risk Assessment version.

Results: In addition to the socioclinical profile revealed, $50.7 \%$ of respondents reported any suicidal ideation, while $6.9 \%$ reported self-injurious behavior without intent to die. Any suicidal and self-injurious behavior was reported by $13.1 \%$ of participants. A total of $71.3 \%$ of respondents reported any recent negative activating events. In addition to any treatment history, observed correlates were hopelessness $(60.7 \%)$, perceived burden on family $(29.5 \%)$, refusing a safety plan (26.1\%), and sexual abuse (11\%). Conversely, reasons for living $(64.9 \%)$, fear of death or dying due to pain and suffering (64.3\%), and spirituality (92\%) were largely endorsed as protective factors. There were multiple significant odds ratios $(P \leq 0.01)$ revealed when independent socioclinical variables were compared with dependent variables in terms of suspected risk and protective factors. In an adjusted logistic regression model, none of the independent variables contributed significantly to any suicidal and self-injurious behavior, any suicidal ideation, or protection from them $(P>0.05)$.

Conclusion: Our preliminary results suggest that there are some socioclinical correlates of any suicide ideation, suicidal and self-injurious behavior, and protection from risky behavior, but which of them contributes significantly to the risk and protective dimensions is yet to be elucidated in prospective community-based studies with larger and more diverse samples.

Keywords: self-injurious behavior, suicidal ideation, risk factors, protective factors, alcohol, drug, abuse

\section{Introduction}

Self-injurious behavior is classified as suicidal or nonsuicidal according to an individual's intent to die or not to die. Self-injurious behavior can be further categorized into complete suicide, a suicide attempt, preparatory acts for imminent suicide behavior, suicide communication, and suicide ideation. The latter events can be further categorized into self-mutilation, parasuicide attempts, and other behavior with no deliberate self-harm. Along this spectrum of suicide events lie indeterminate behaviors broadly categorized into self-injurious behavior with suicidal intent unknown and with not enough information. ${ }^{1}$ Despite comprehensive exploratory probes and extensive
Correspondence: Naseem Akhtar Qureshi Division of Studies and Research, General Directorate of Research and Studies, Sulaimania Medical Complex, Ministry of Health, Riyadh II I76, Kingdom of Saudi Arabia

Tel +9660 I4735038

Fax +96 $60 \quad 14735039$

Email qureshinaseem@live.com 
research, many difficulties tend to cloak the issue of exact categorization of suicide and parasuicide behavior, including accidental events and their associated sociocultural and pathological functions in the light of automatic and social reinforcement. ${ }^{2}$

Mental disorders have a close relationship with selfinjurious behavior, and $50 \%$ or more of all people who die by suicide have at least one mental illness, especially depression or substance abuse problems. ${ }^{3}$ There are one million deaths by suicide each year, representing $1.8 \%$ of the total global burden of disease, and half of them have a history of suicide attempts attributed to multiple risk factors. ${ }^{4}$ About 20 million people self-harm nonfatally, and add substantial burden to the health care system. Notably, $10 \%$ of those with parasuicide attempts guided by psychological motives and meaningfulness compatible with a functionalist approach also eventually die from suicide. ${ }^{5,6}$ A large Western database has revealed multiple risk factors underlying self-injurious behavior and substance abuse, which among others, include coexisting mental and physical disorders, impulsivity, problem drinking, partner relationship problems, significant life stressors and events, previous suicide attempts, and lack of social and family support. ${ }^{7-22}$ Furthermore, level of acculturation, cultural conflicts, and stigma can also influence the likelihood of self-injurious behavior. ${ }^{23}$ From the known risk factors for suicidal behavior, several investigators have developed 10 behavior patterns that need to be closely monitored, which are: talking or in any other way communicating a willingness to die or kill oneself; substance abuse; expressing a sense of purposelessness; showing signs of anxiety, including agitation and changes in sleep patterns; expressing feelings of being trapped in various personal situations; expressing feelings of hopelessness; withdrawing from social activities with friends and family; showing unusual signs of anger, engaging in reckless behavior; and exhibiting signs of mood changes. ${ }^{24,25}$ On the other hand, factors reported to be protective against self-injurious behavior include reasons for living, sense of responsibility to family or others, supportive family and social networks, fear of death or dying due to pain and suffering, spirituality, work engagement, and continuing treatment of underlying mental and physical disorders. ${ }^{26-31}$

In contrast, there is a meager database on self-injurious behavior in the Middle Eastern world. In a retrospective study of attempted suicides $(n=365)$ admitted to the National Guard Hospital, Riyadh, Saudi Arabia, it was shown that the two most important reasons for attempting suicide included family/marital problems and mood disorders among females and males, respectively. ${ }^{32}$ Notably, alcohol and/or drug abuse is a major contributor to the morbidity and mortality of selfinjurious behavior worldwide.

To our knowledge, there is no study that has estimated the prevalence or identified risk and protective factors for self-harm behavior among patients with alcohol or drug abuse problems in the Kingdom of Saudi Arabia. Therefore, this research aimed to assess the magnitude of suicide ideation, attempts, and parasuicidal behavior, and to explore possible risk and protective factors among patients diagnosed with alcohol or drug abuse. We hypothesized that the prevalence rate of self-harm behavior would be relatively low in Saudi culture, and attributable to a protective sociocultural belief system.

\section{Materials and methods}

A convenience sample of 736 patients with a diagnosis of alcohol or drug abuse was recruited proportional to the bed capacity of three settings, ie, the Al-Amal complex for mental health in Riyadh $(\mathrm{n}=250)$, Dammam $(\mathrm{n}=250)$, and Al-Amal, Jeddah $(\mathrm{n}=236)$. We included patients aged $\geq 16$ years and $<60$ years who were second or third consecutive outpatients and inpatients seen on all working days of the week who fulfilled the Diagnostic and Statistical Manual of Mental Disorders, Fourth Edition, Text Revision (DSM-IV) criteria for substance use disorders with or without a history of suicidal or deliberate self-harm behavior. DSM-IV criteria are structured and research-oriented, but cross-sectional interviews conducted by qualified psychiatrists having indepth knowledge of the DSM-IV were unstructured. DSM-IV describes a variety of "substance use disorders" and this research focuses on alcohol or drug abuse, because a patient with a substance use disorder has passed the stages of substance use to abuse, and the relevant literature suggests that these stages keep on shifting in both directions, ie, from use or abuse to addiction and vice versa in the patient's life trajectory. Other inclusion criteria were: alcohol or drug abuse comorbid with other DSM-IV mental disorders; a history of repeated self-harm with and without intent to die; and patients with alcohol or drug abuse and coexisting medical problems not causing psychosis. Comorbid diagnosis of medical disorders was based on patient self-reporting. Patients with brain injury and those with psychoses caused by medical and neurological conditions were excluded from this study.

Following a detailed interview of all eligible patients together with a review of their medical files, sociodemographic and clinical variables were abstracted on a semistructured sheet. Primary and comorbid diagnoses were based on medical file reviews and interviews conducted 
by assistant researchers. The respondents were arbitrarily categorized into four income groups, based on total annual income in Saudi Riyals (SR) whereby 1 USD is equivalent to $3.75 \mathrm{SR}$ (low $\leq 24,000 \mathrm{SR}$, middle income 25,000-50,000 SR, high income $>51,000-99,000 \mathrm{SR}$, and wealthy income $>100,000 \mathrm{SR})$. The Columbia Suicide Severity Scale (CSSS) Risk Assessment version was used for assessing suicidal behavior. ${ }^{31}$ Low-income and middle-income groups were pooled for statistical purposes and considered as the low-income group. This scale has good reliability and validity, and assesses actual attempts, nonsuicidal selfinjurious behavior, interrupted attempts, aborted attempts, preparatory acts or behaviors, absence of suicidal behavior, frequency of suicidal ideations over the last one week, and risk factors for and protective factors against suicide behavior. Bilingual interviewers with an MD/MS qualification read (often both in Arabic and English languages) the probes from this scale and definitions of each sub-item of six domains slowly and clearly to the respondents, and their responses were noted by the interviewers on scale templates. From an ethical perspective, the complete research proposal including informed consent was submitted to the General Administration for Medical Research, Ministry of Health. This department has both scientific and ethical committees for evaluating submitted research protocols. Finally, permission was obtained for conducting this research in the Ministry of Health setting. All participants were requested to sign the informed consent.

\section{Data analysis}

The data collected were entered into a computer and cleaned before conducting the analysis. In addition to calculating the frequency distribution and statistics of the variables, odds ratios were estimated by comparing certain socioclinical and risk factors. Odds ratio estimates describe the strength of association or nonindependence between two binary variables. We have reported an odds ratio reflecting a significant association between two variables. A $P$ value $\leq 0.01$ was considered to be statistically significant. Adjusted regression analysis was also performed to elucidate the most significant protective factors and predictors of suicide and self-injurious behavior. The Statistical Package for Social Sciences version 10 (SPSS Inc, Chicago, IL) was used to analyze the data.

\section{Results}

\section{Sociodemographic variables}

All the respondents were male (substance abuse is uncommon among women in Saudi Arabia). The mean age of the respondents was $32.3 \pm 9.2$ (range 16-60) years. The majority were Saudis $(97.4 \%$ ), with $66.2 \%$ being unemployed and $25.3 \%$ being illiterate. Fifty-nine percent of the respondents were single, $58.2 \%$ were living in families, and $49.3 \%$ reported being on a middle income. The participants were drawn equally from urban and rural areas (Table 1).

\section{Clinical variables}

As shown in Table 2, the majority of the respondents (98.2\%) were smokers, and $95.3 \%$ were chronic smokers (more than 6 months). The respondents were also chronic abusers of multiple addictive substances, including amphetamines (39.5\%), alcohol (19.7\%), heroin (17.4\%), cannabis (16.3\%),

Table I Selected characteristics of patients with alcohol or drug abuse $(n=736)$

\begin{tabular}{|c|c|c|}
\hline Variable & $\mathbf{n}$ & $\%$ \\
\hline \multicolumn{3}{|l|}{ Age, years } \\
\hline $15-24$ & 160 & 21.7 \\
\hline $25-34$ & 313 & 42.6 \\
\hline $35-44$ & 164 & 22.2 \\
\hline $45-54$ & 92 & 12.5 \\
\hline$>55$ & 7 & 1.0 \\
\hline \multicolumn{3}{|l|}{ Gender } \\
\hline Male & 736 & 100.0 \\
\hline \multicolumn{3}{|l|}{ Nationality } \\
\hline Saudi & 717 & 97.4 \\
\hline Not Saudi & 19 & 2.6 \\
\hline \multicolumn{3}{|l|}{ Education } \\
\hline Primary & 107 & 14.5 \\
\hline Secondary & 104 & 14.1 \\
\hline Intermediate & 303 & 41.2 \\
\hline College & 36 & 4.9 \\
\hline Illiterate & 186 & 25.3 \\
\hline \multicolumn{3}{|l|}{ Employment } \\
\hline Employed & 249 & 33.8 \\
\hline Unemployed & 487 & 66.2 \\
\hline \multicolumn{3}{|l|}{ Marriage status } \\
\hline Single & 437 & 59.4 \\
\hline Married & 216 & 29.3 \\
\hline Others & 83 & 11.3 \\
\hline \multicolumn{3}{|l|}{ Family types } \\
\hline Joint & 428 & 58.2 \\
\hline Nuclear & 308 & 41.8 \\
\hline \multicolumn{3}{|l|}{ Income level } \\
\hline Low & 241 & 32.7 \\
\hline Middle & 363 & 49.3 \\
\hline High & 94 & 12.8 \\
\hline Very wealthy & 38 & 5.2 \\
\hline \multicolumn{3}{|l|}{ Residence status } \\
\hline Rural & 370 & 50.3 \\
\hline Urban & 355 & 48.2 \\
\hline Nomads & 11 & 1.5 \\
\hline \multicolumn{3}{|l|}{ Religion } \\
\hline Muslim (Islam) & 733 & 99.6 \\
\hline Non-Muslim (non-Islam) & 3 & 0.4 \\
\hline
\end{tabular}


Table 2 Substance use and comorbid conditions among patients with alcohol or drug abuse $(n=736)$

\begin{tabular}{|c|c|c|}
\hline Variables & $\mathbf{n}$ & $\%$ \\
\hline \multicolumn{3}{|c|}{ Cigarette smoking } \\
\hline Yes & 723 & 98.2 \\
\hline No & 13 & 1.8 \\
\hline \multicolumn{3}{|c|}{ Duration of cigarette smoking } \\
\hline$<6$ months & 21 & 2.9 \\
\hline$>6$ months & 702 & 95.3 \\
\hline 0 months & 13 & 1.8 \\
\hline \multicolumn{3}{|c|}{ Pattern of drug abuse } \\
\hline Alcohol & 145 & 19.7 \\
\hline Cannabis & 120 & 16.3 \\
\hline Heroin & 128 & 17.4 \\
\hline Amphetamines & 291 & 39.5 \\
\hline Cocaine & 13 & 1.8 \\
\hline Volatile substances & 8 & 1.1 \\
\hline Others & 31 & 4.2 \\
\hline \multicolumn{3}{|c|}{ Duration of drug abuse } \\
\hline$<6$ months & 9 & 1.2 \\
\hline$>6$ months & 727 & 98.8 \\
\hline \multicolumn{3}{|c|}{ Comorbid mental disorder } \\
\hline Anxiety & 127 & 17.3 \\
\hline Depression & 419 & 56.9 \\
\hline Others & 44 & 6.0 \\
\hline None & 146 & 19.8 \\
\hline \multicolumn{3}{|c|}{ Duration of mental disorder* } \\
\hline$<6$ months & 22 & 3.0 \\
\hline$>6$ months & 568 & 77.2 \\
\hline 0 months & 146 & 19.8 \\
\hline \multicolumn{3}{|c|}{ Comorbid physical disorder } \\
\hline Diabetes mellitus & 12 & 1.6 \\
\hline Hypertension & 8 & I.I \\
\hline Others & 62 & 8.4 \\
\hline None & 654 & 88.9 \\
\hline \multicolumn{3}{|c|}{ Duration of physical disorders* } \\
\hline$<6$ months & 3 & 0.4 \\
\hline$>6$ months & 79 & 10.7 \\
\hline 0 months & 654 & 88.9 \\
\hline
\end{tabular}

Note: *Coexisting disorders.

and others. Overall, 98.8\% reported drug abuse for a period of more than 6 months. Depression was the most prevalent comorbid mental diagnosis $(56.9 \%)$ in respondents, followed by anxiety disorders (17.3\%). Approximately $11 \%$ of respondents had a coexisting physical disorder (eg, diabetes mellitus, hypertension).

\section{CSSS Risk Assessment version}

The CSSS Risk Assessment version includes six domains, ie, suicidal and self-injury behavior, suicide ideation, recent activating events, treatment history, recent clinical status, and recent protective factors. Several forms of suicidal behavior were reported by $6.2 \%$ of respondents, while $6.9 \%$ reported self-injurious behavior without suicide intent (Table 3). Suicidal ideation items were endorsed by $50.7 \%$ of participants; $71.3 \%$ of patients reported activating negative events; any treatment history items were endorsed by $93.3 \%$ of patients. Furthermore, patients' reports on the clinical items included hopelessness (60.7\%), highly

Table 3 Descriptive results from Columbia Suicide Severity Rating Scale Risk Assessment version among patients with alcohol or drug abuse $(\mathrm{n}=736)$

\begin{tabular}{|c|c|c|}
\hline Variables & $\mathbf{n}$ & $\%$ \\
\hline \multicolumn{3}{|l|}{ Suicidal and self-injury behavior* } \\
\hline Actual suicidal attempt - yes & 20 & 2.7 \\
\hline Interrupted attempt - yes & 16 & 2.2 \\
\hline Aborted attempt - yes & 7 & 1.0 \\
\hline Other preparatory acts to kill self - yes & 2 & 0.3 \\
\hline Self-injury behavior without suicide intent - yes & 51 & 6.9 \\
\hline Any suicidal and self-injury behavior & 96 & 13.1 \\
\hline \multicolumn{3}{|l|}{ Suicide ideation* } \\
\hline Wish to be dead - yes & 273 & 37.1 \\
\hline Suicidal thoughts - yes & 80 & 10.9 \\
\hline Suicidal thoughts with methods $* *-$ yes & 10 & 1.4 \\
\hline Suicidal intent without specific plan - yes & 2 & 0.3 \\
\hline Suicidal intent with specific plan - yes & 7 & 1.0 \\
\hline Any suicide ideation & 372 & 50.7 \\
\hline \multicolumn{3}{|l|}{ Recent activating events* } \\
\hline Recent loss or other significant negative event - yes & 247 & 33.6 \\
\hline Pending incarceration or homelessness - yes & 171 & 23.2 \\
\hline Current or pending isolation or feeling alone - yes & 254 & 34.5 \\
\hline Any recent activating events & 672 & 71.3 \\
\hline \multicolumn{3}{|l|}{ Treatment history* } \\
\hline Previous psychiatric diagnosis and treatments - yes & 20 & 2.7 \\
\hline Hopelessness or dissatisfied with treatment - yes & 365 & 49.6 \\
\hline Noncompliant with treatment - yes & 253 & 34.3 \\
\hline Not receiving treatment - yes & 49 & 6.7 \\
\hline Any treatment history & 687 & 93.3 \\
\hline \multicolumn{3}{|l|}{ Recent clinical status* } \\
\hline Hopelessness - yes & 447 & 60.7 \\
\hline Major depressive episode - yes & 58 & 7.9 \\
\hline Mixed affective episode - yes & 3 & 0.4 \\
\hline Command hallucinations to hurt self - yes & 2 & 0.3 \\
\hline Highly impulsive behavior - yes & 60 & 8.2 \\
\hline Agitation or severe anxiety - yes & 40 & 5.4 \\
\hline Perceived burden on family or others - yes & 217 & 29.5 \\
\hline Chronic physical pain/acute medical condition - yes & 32 & 4.3 \\
\hline Homicidal ideation - yes & 29 & 3.9 \\
\hline Aggressive behavior towards others - yes & 44 & 6.0 \\
\hline Method for suicide available - yes & 19 & 2.6 \\
\hline Refuses or feels unable to agree to safety plan - yes & 192 & 26.1 \\
\hline Sexual abuse - yes & 81 & 11.0 \\
\hline Family history of suicide - yes & 2 & 0.3 \\
\hline \multicolumn{3}{|l|}{ Recent protective factors* } \\
\hline Identifies reasons for living - yes & 478 & 64.9 \\
\hline Responsibility to family or others: living with family - yes & 153 & 20.8 \\
\hline Supportive social network or family - yes & 265 & 36.0 \\
\hline Fear of death or dying due to pain and suffering - yes & 473 & 64.3 \\
\hline Belief that suicide is immoral, highly spirituality - yes & 678 & 92.1 \\
\hline Engaged in work or school - yes & 120 & 16.3 \\
\hline
\end{tabular}

Notes: *Mutually exclusive categories; ***without specific plan or intent to act. 
impulsive behavior (8.2\%), a perceived burden on family (29.5\%), refusal or feeling unable to agree to a safety plan (26.1\%), and sexual abuse (11.0\%). With regard to protective factors, the majority of respondents observed that there were reasons for living $(64.9 \%)$, showed interest in family or others $(20.8 \%)$, had supportive social networks or family ties $(36.0 \%)$, had a fear of death or dying due to pain and suffering $(64.3 \%)$, were engaged in work or school (16.3\%), and held the belief that suicide is immoral (92.1\%).

\section{Associations between CSSS Risk Assessment version, demographics, and comorbidity}

Table 4 summarizes associations (odds ratios) between the characteristics of the CSSS Risk Assessment version and demographic and comorbid variables. These odds ratios are unadjusted results. Because of multiple comparisons, variables with $P<0.01$ are reported. Suicide ideation was reported three times more often by participants with high incomes who were from an urban background. Negative activating events were three times more commonly associated with low education, low income, rural background, and comorbid mental disorders. Furthermore, any treatment history was three times more likely to be associated with a low income and a rural background. A variety of clinical variables, in terms of hopelessness, depression, family burden, chronic physical diseases, homicidal ideation, and sexual abuse, were more frequently associated with age over 30 years, low income and education, rural background, and coming from a single family (a married couple living independently).

With regard to recently protective factors, identification of reasons for living was 1.5 times more common among adults (age $>30$ years), and 2.5 and 4.0 times more common among those with no job and those from a rural background, respectively. Responsibility to family or others and living with family was twice as common among participants with no employment, while a supportive social network or family was about 1.5 times more common among those with no employment and no cooccurring medical illnesses, respectively. Furthermore, fear of death or dying with pain and suffering was about 3.0 and 2.0 times more common among participants in the rural and lowincome groups, respectively. Being engaged in work or school was a strong protective factor against suicidal behavior.

We included several independent variables in an adjusted logistic regression model in terms of age, education, family type, residence status, nationality, income level, marital status, employment, religion, cigarette smoking, and comorbid mental and physical disorders. We also included recent protective factors as dependent variables. The computed average of each one of the three dependent variables (any suicidal and self-injurious behavior, any suicide ideation, and protective factors) were separately recoded into binary variables. Surprisingly, none of the independent variables regressed against binary dependent variables survived the logistic regression analysis $(<0.05)$. The probable reasons for this are mentioned in the discussion.

\section{Discussion}

According to this hospital-based study, the majority of patients with drug abuse, suicidal ideation, and suicidal and self-harm behavior were young adults with minimal education. Just over half of the patients were single, unemployed, and were from joint families (a married couple living with his/or her parents). Furthermore, half of the patients were from a rural background and about one third had a low income. Because we had only one local study available on this subject, we compared our results with those of studies in alcohol/drug abuse/addiction populations from the Western world. The researchers feel that such comparisons are limited in many ways, and especially from the cultural perspective. Our sociodemographic profile of an addictive population with suicidal and self-injurious behavior is consistent with that reported in other studies. ${ }^{5,32,34}$ A similar demographic trend was also found in our previous addiction research that did not assess suicide behavior. An important implication of this study is that alcohol and drug abuse coupled with selfinjurious behavior has already spread to rural communities. In addition to residential status and family composition, other sociodemographic variables, ie, young age, single marital status, unemployment, and low income, have been identified as possible risk factors for suicidal communication, ideation, and

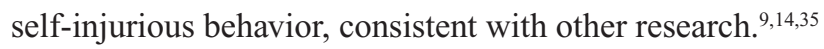

In our study, the majority of patients were chronic smokers, stimulant abusers, and had comorbid anxiety disorders and depression, but less physical diseases and this clinical trend is mostly consistent with other studies. ${ }^{34}$ In our two studies of psychiatric patients with trihexiphenidyl abuse, a similar pattern of smoking was also found. ${ }^{36,37}$

Any suicide ideation and any suicidal and self-injurious behavior without intent to die were reported by about $50.7 \%$ and $13.1 \%$ of respondents, respectively. These prevalence rates, as revealed by the CSSRS Risk Assessment version in psychiatric outpatients and inpatients with a history of drug abuse, are presumably at variance with the findings of studies of community samples from other cultures. ${ }^{38}$ In a Chinese study, Cheng et al found that $17.4 \%$ of students 
Table 4 Associations of characteristics of Columbia Suicide Severity Rating Scale Risk Assessment version with demographic and comorbid variables

\begin{tabular}{|c|c|c|c|c|}
\hline \multirow{2}{*}{$\begin{array}{l}\text { Characteristics } \\
\text { Suicidal ideation }\end{array}$} & \multicolumn{2}{|c|}{ Demographic and comorbid variables } & \multirow[t]{2}{*}{ OR } & \multirow[t]{2}{*}{$95 \% \mathrm{Cl}$} \\
\hline & Income & & & \\
\hline & High & Low & & \\
\hline & Yes, n (\%)* & Yes, n (\%)* & & \\
\hline Wish to be dead & 354 (48.09) & $250(33.97)$ & 3.347 & $2.075-5.398$ \\
\hline \multirow[t]{3}{*}{ Suicidal thoughts } & $530(72.01)$ & $74(10.05)$ & 2.932 & $1.248-6.890$ \\
\hline & Residence & & & \\
\hline & Urban & Rural & & \\
\hline Wish to be dead & $328(44.57)$ & 234 (31.79) & 12.89 & $8.72-19.077$ \\
\hline \multirow[t]{2}{*}{ Suicidal thoughts } & $328(44.57)$ & $53(7.20)$ & 1.963 & $1.205-3.198$ \\
\hline & Educational level & & & \\
\hline Activating events & Intermediate and higher & Secondary or less & & \\
\hline Recent loss or other significant negative event & $90(12.23)$ & $157(21.33)$ & 1.810 & $1.322-2.477$ \\
\hline \multirow[t]{3}{*}{ Current or pending isolation or feeling alone } & $100(13.59)$ & $154(20.92)$ & 1.515 & $1.112-2.062$ \\
\hline & Income & & & \\
\hline & High & Low & & \\
\hline Pending incarceration or homelessness & $12(1.63)$ & $159(21.60)$ & 3.573 & $1.921-6.645$ \\
\hline \multirow[t]{3}{*}{ Current or pending isolation or feeling alone } & $33(4.48)$ & $221(30.03)$ & 1.731 & $1.129-2.654$ \\
\hline & Residence & & & \\
\hline & Urban & Rural & & \\
\hline \multirow[t]{3}{*}{ Pending incarceration or homelessness } & $13(1.77)$ & I $58(2 \mid .47)$ & 18.64 & $10.33-33.63$ \\
\hline & Comorbid mental disorde & & & \\
\hline & No & Yes & & \\
\hline Pending incarceration or homelessness & $22(2.99)$ & $149(20.24)$ & 1.904 & $1.167-3.108$ \\
\hline \multirow[t]{2}{*}{ Treatment history } & Income & & & \\
\hline & High & Low & & \\
\hline Hopeless or dissatisfied with treatment & $32(4.35)$ & $333(45.24)$ & 3.840 & $2.500-5.898$ \\
\hline \multirow[t]{2}{*}{ Noncompliant with treatment. } & $35(4.76)$ & $218(29.62)$ & 1.565 & $1.028-2.384$ \\
\hline & Urban & Rural & & \\
\hline Hopeless or dissatisfied with treatment & $117(15.90)$ & $248(33.70)$ & 3.793 & $2.794-5.148$ \\
\hline Not receiving treatment & II ( 1.49$)$ & $38(5.16)$ & 3.465 & $1.742-6.890$ \\
\hline \multirow[t]{2}{*}{ Clinical status (recent) } & Income & & & \\
\hline & High & Low & & \\
\hline \multirow[t]{2}{*}{ Hopelessness } & 47 (6.39) & $400(54.35)$ & 3.546 & $2.391-5.259$ \\
\hline & Urban & Rural & & \\
\hline \multirow[t]{3}{*}{ Hopelessness } & $160(21.74)$ & $267(36.28)$ & 3.721 & $2.72 I-5.089$ \\
\hline & Comorbid physical illness & & & \\
\hline & No & Yes & & \\
\hline Major depressive episode & $46(6.25)$ & $12(1.63)$ & 2.266 & $1.146-4.480$ \\
\hline \multirow[t]{2}{*}{ Mixed affective episode } & I $(0.12)$ & $2(0.27)$ & 16.33 & $1.46-182.06$ \\
\hline & Intermediate and high & Secondary or less & & \\
\hline Perceived burden on family or others & $74(10.05)$ & $143(19.43)$ & 2.016 & $1.45 \mathrm{I}-2.802$ \\
\hline \multirow[t]{3}{*}{ Refuses or feels unable to agree to safety plan } & $72(9.78)$ & $120(16.30)$ & 1.606 & $1.147-2.250$ \\
\hline & Comorbid physical illness & & & \\
\hline & No & Yes & & \\
\hline \multirow{2}{*}{$\begin{array}{l}\text { Chronic physical pain or other acute } \\
\text { medical problem }\end{array}$} & $5(0.68)$ & $27(3.67)$ & 63.72 & $23.6-172.02$ \\
\hline & Urban & Rural & & \\
\hline \multirow[t]{2}{*}{ Homicidal ideation } & $3(0.36)$ & $26(3.53)$ & 2.578 & 28.65 \\
\hline & $\leq \mathbf{3 0}$ years & $>30$ years & & \\
\hline \multirow[t]{2}{*}{ Sexual abuse (lifetime) } & $14(1.90)$ & $67(9.10)$ & 5.294 & $2.917-9.607$ \\
\hline & Joint family & Single family & & \\
\hline Sexual abuse (lifetime) & $14(1.90)$ & $67(9.10)$ & 3.686 & $2.03 I-6.692$ \\
\hline Protective factors (recent) & $\leq \mathbf{3 0}$ years & $>30$ years & & \\
\hline Identifies reasons for living & $218(29.62)$ & $260(35.33)$ & 1.415 & $1.044-1.918$ \\
\hline
\end{tabular}


Table 4 (Continued)

\begin{tabular}{|c|c|c|c|c|}
\hline \multirow[t]{2}{*}{ Characteristics } & \multicolumn{2}{|c|}{ Demographic and comorbid variables } & \multirow[t]{2}{*}{ OR } & \multirow[t]{2}{*}{$95 \% \mathrm{Cl}$} \\
\hline & Employed & Unemployed & & \\
\hline Identifies reasons for living & $196(26.63)$ & $282(38.32)$ & 2.688 & I.889-3.825 \\
\hline Responsibility to family or others; living with family & $82(11.14)$ & $71(9.65)$ & 2.877 & $1.997-4.144$ \\
\hline Supportive social network or family & $107(14.54)$ & I58 (2I.47) & 1.569 & $1.146-2.149$ \\
\hline \multirow[t]{2}{*}{ Engaged in work or school } & $109(14.81)$ & II (I.49) & 33.69 & $|7.62-64.4|$ \\
\hline & Urban & Rural & & \\
\hline Identifies reasons for living & $175(23.78)$ & $303(41.17)$ & 3.996 & $2.889-5.527$ \\
\hline \multirow[t]{3}{*}{ Fear of death or dying due to pain and suffering } & $184(25.0)$ & $289(39.27)$ & 2.919 & $2.133-3.955$ \\
\hline & Income & & & \\
\hline & High & Low & & \\
\hline \multirow[t]{3}{*}{ Fear of death or dying due to pain and suffering } & $59(8.02)$ & $4 \mid 4(56.25)$ & 2.696 & I.837-3.957 \\
\hline & Comorbid & & & \\
\hline & No & Yes & 1.718 & I.082-2.727 \\
\hline Supportive social network or family & $226(30.70)$ & $39(5.30)$ & & \\
\hline
\end{tabular}

Notes: 30 years as cutoff point was based on arbitration. *Subsequent sociodemographic and comorbid variables with responses yes, number (\%). Abbreviations: $\mathrm{Cl}$, confidence interval; $\mathrm{OR}$, odds ratio.

had seriously considered attempting suicide, and 8.1\% had made a specific plan for suicide during the 12 months preceding the survey. ${ }^{39}$ In a Turkish study, $23 \%$ of students (aged 13-18 years) reported having had thoughts of killing themselves during the previous 12 months or during their lifetime. ${ }^{40}$ The reported rate of suicidal and self-injurious behavior would presumably be much less if such study was conducted in the general population, as was found in recent US surveys of youth. ${ }^{38,41}$ In a drug abuse population, reported rates of lifetime suicide attempts have been much higher at $47 \%$, and this figure was higher in polysubstance abusers $(58 \%)$ than in alcoholics $(38 \%) .^{42}$ In another study of 80 opiate addicts, the lifetime prevalence rate of self-injurious behavior was $49 \%{ }^{43}$ Notably, the prevalence of suicidal and self-injurious behavior is highly variable, which is attributable to a variety of factors, including sample characteristics, measurement tools, and study design. The epidemiological trend of variable suicidal and self-injurious behavior in the present study might be attributed to sociocultural factors.

Notably, several significant odds ratios were found for socioclinical variables and risk and protection factors when sociodemographic variables and risk/protective factors were compared, and overall revealed significant results which are mostly consistent with the world literature on risk and protective factors concerning suicidal behavior. ${ }^{7-22,44,45}$ Certain trends in suicide ideation and self-injurious behavior were identified in this study, and are summarized because of their sociocultural significance; respondents had a three-fold lower risk of suicidal behavior if they had families and a history of sexual or physical abuse. The coupling of sexual abuse and minimal suicide behavior is surprising and needs investigation in larger groups of patients with a history of drug and sexual abuse. We also found that participants were 18 times more at risk of suicidal behavior when they were awaiting imprisonment or were from rural areas. A combination of drug abuse, crime, a rural background, and suicidal communication is apparently emerging in our culture; participants were six times more vulnerable to suicidal behavior if they were younger and had a family history of violence; they were four times more at risk of suicide and self-injurious behavior if they were single and had a family history of violence; were 2-4 times more at risk for suicidal behavior if they had a low income, were unmarried, lived in joint families, came from a rural background, or suffered from depression; were 64 times more at risk of suicidal behavior when they suffered from chronic pain or comorbid medical illness than those without medical disorders.

Factors identified to be protective against suicidal behavior need emphasis. Family connectedness, as found in joint families, has been reported to be a protective factor against suicidal behavior among adolescents with a history of sexual abuse. ${ }^{46}$ In another study of the role of psychological buffers in the development of hopelessness and suicidal ideation, the authors found that life satisfaction, self-esteem, perception of family cohesion, and social support were mitigating factors against hopelessness and suicidal ideation, respectively. ${ }^{47}$ Multiple aches and pains commonly reported by the population in the Eastern world, also found in this study, have sociocultural explanatory models. Although there is a huge amount of research data on risk factors for suicide and self-injurious behavior, relatively little is said about protective factors against suicidal behavior. ${ }^{48}$ According to this study, 
multiple possible factors protective against suicide behaviors were consistent with those identified in other studies. ${ }^{41,45,48}$ In addition, several studies have found other protective factors against suicide and self-injurious behavior. ${ }^{40,49}$ In our study, these protective factors were apparently ineffective in about $50 \%$ of cases as far as suicide ideation and communication were concerned, but were very effective against carrying out thoughts about suicide and parasuicide attempts. How risk factors for and protective mechanisms against suicide behavior interact to manifest in either of the possible outcomes, ie, suicidal or no suicidal behavior, is challenging to investigate and limited information is available. ${ }^{50}$ Moreover, whether both protective factors against and risk factors for suicide and self-injurious behavior are the same or different is debatable and challenging to investigate. , $^{6,51,52}$

This is the first cross-sectional study in a hospital-based population that has attempted to estimate the prevalence of suicidal and self-injurious behavior and associated risk and protective factors. However, its tentative preliminary results cannot be generalized to a larger population and need to be interpreted cautiously. The study has some limitations. Sample selection was not randomized and there was no determination of an adequate sample size, with recruitment being merely proportional to the bed capacity at each setting. Further, there was no consideration of inter-rater differences, although all coinvestigators were $\mathrm{MD} / \mathrm{MS}$ graduates with considerable clinical experience, and were intensively briefed by the principal investigators about the rating scale in two mini-workshops. This scale has good reliability and validity and has been used extensively worldwide, although no attempt was made to assess its statistical properties in relation to the Saudi setting. Furthermore, our results are descriptive only and require confirmation in a larger and more diverse sample to understand suicidal behavior better. The results are useful as preliminary data for informing further research or generating hypotheses for further testing, but they do not reflect definite "risk or protective factors" because they are descriptive only. Surprisingly, the adjusted logistic regression analysis did not yield significant results. Our negative results might be attributed to measurement error, our small hospital-based sample, and/or more negative responses on any suicide ideation and suicidal and self-injurious behavior and more positive responses on protective items in the rating scale, and others.

In summary, rates of any suicide ideation or suicidal and self-injurious behavior are relatively low in this study. The protective factors identified might possibly act against translating suicidal ideation into suicide attempts and selfinjurious behavior. Prospective community-based studies are needed to elucidate the most significant protective factors and predictors of suicide ideation, or suicidal and self-injurious behavior in the future.

\section{Acknowledgment}

The authors thank King Abdulaziz City for Science and Technology for financially supporting this research (Grant 12-19) and to Professor Assaf Al-Assaf of Oklahoma University, USA for statistical help.

\section{Disclosure}

NAQ and AA-H are members of the ethical committee of the General Administration for Research and Studies the former is affiliated with this administration.

\section{References}

1. Posner K, Oquendo MA, Gould M, Stanley B, Davies M. Columbia Classification Algorithm of Suicide Assessment (C-CASA): classification of suicidal events in the FDA's pediatric suicidal risk analysis of antidepressants. Am J Psychiatry. 2007;164:1035-1043.

2. Nock MK. Actions speak louder than words: an elaborated theoretical model of the social functions of self-injury and other harmful behaviors. Appl Prev Psychol. 2008;12:159-168.

3. Mann JJ. A current perspective of suicide and attempted suicide. Ann Intern Med. 2002;136;302-311.

4. World Health Organization. Suicide prevention. Available at: http:// www.who.int/mental_health/prevention/suicide/suicideprevent/en/. Accessed June 10, 2012.

5. Mitchell AJ, Dennis M. Self-harm and attempted suicide in adults: 10 practical questions and answers for emergency department staff. Emerg Med J. 2006;23:251-255.

6. Claes L, Vandereycken W. Self-injurious behavior: differential diagnosis and functional differentiation. Compr Psychiatry. 2007;48:137-144.

7. World Health Organization. Mental and Neurological Disorders. Factsheet 265. Factsheet No 265 2001. Indian J Med Sci. 2002;56: 25-29.

8. Foster T, Gillespie K, McClelland R. Mental disorders and suicide in Northern Ireland. Br J Psychiatry. 1997;170:447-452.

9. Shaffer D, Craft L. Methods of adolescent suicide prevention. J Clin Psychiatry. 1999;60 Suppl 2:70-74.

10. Brausch AM, Gutierrez PM. The role of body image and disordered eating as risk factors for depression and suicidal ideation in adolescents. Suicide Life Threat Behav. 2009;39:58-71.

11. Foley DL, Goldston DB, Costello EJ, Angold A. Proximal psychiatric risk factors for suicidality in youth: the Great Smoky Mountains Study. Arch Gen Psychiatry. 2006;63:1017-1024.

12. Russell ST, Joyner K. Adolescent sexual orientation and suicide risk: evidence from a national study. Am J Public Health. 2001;91: 1276-1281.

13. Boden JM, Fergusson DM, Horwood LJ. Anxiety disorders and suicidal behaviors in adolescence and young adulthood: findings from a longitudinal study. Psychol Med. 2007;37:431-440.

14. Dube SR, Anda RF, Felitti VJ, Chapman DP, Williamson DF, Giles WH. Childhood abuse, household dysfunction, and the risk of attempted suicide throughout the life span: findings from the Adverse Childhood Experiences Study. JAMA. 2001;286:3089-3096.

15. Brown J, Cohen P, Johnson JG, Smailes EM. Childhood abuse and neglect: specificity of effects on adolescent and young adult depression and suicidality. J Am Acad Child Adolesc Psychiatry. 1999;38:1490-1496.

16. McKeown RE, Garrison CZ, Cuffe SP, Waller JL, Jackson KL, Addy CL. Incidence and predictors of suicidal behaviors in a longitudinal sample of young adolescents. J Am Acad Child Adolesc Psychiatry. 1998;37:612-619. 
17. Remafedi G. Suicidality in a venue-based sample of young men who have sex with men. J Adolesc Health. 2002;31:305-310.

18. Oquendo MA, Mann JJ. The biology of impulsivity and suicidality. Psychiatr Clin North Am. 2000;23:11-25.

19. Gould MS, King R, Greenwald S, et al. Psychopathology associated with suicidal ideation and attempts among children and adolescents. J Am Acad Child Adolesc Psychiatry. 1998;37:915-923.

20. Shah S, Hoffman RE, Wake L, Marine WM. Adolescent suicide and household access to firearms in Colorado: results of a case-control study. J Adolesc Health. 2000;26:157-163.

21. Beautrais AL, Joyce PR, Mulder RT. Precipitating factors and life events in serious suicide attempts among youths aged 13 through 24 years. J Am Acad Child Adolesc Psychiatry. 1997;36:1543-1551.

22. Heru AM, Stuart GL, Recupero PR. Family functioning in suicidal inpatients with intimate partner violence. Prim Care Companion J Clin Psychiatry. 2007;9:413-418.

23. Ahmed K, Mohan RA, Bhugra D. Self-harm in South Asian women a literature review informed approach to assessment and formulation. Am J Psychother. 2007;61:71-81.

24. Rudd MD, Berman AL, Joiner TE Jr, et al. Warning signs for suicide theory, research, and clinical applications. Suicide Life Threat Behav. 2006;36:255-262.

25. Wintersteen MB, Diamond GS, Fein JA. Screening for suicide risk in the pediatric emergency and acute care setting. Curr Opin Pediatr. 2007;19:398-404.

26. Taliaferro LA, Rienzo BA, Pigg RM, Miller MD, Dodd VJ. Spiritual well-being and suicidal ideation among college students. $J$ Am Coll Health. 2009;58:83-90.

27. Lizardi D, Currier D, Galfalvy H, et al. Perceived reasons for living at index hospitalization and future suicide attempt. J Nerv Ment Dis. 2007;195:451-455.

28. Fitzpatrick KM, Piko BF, Miller E. Suicide ideation and attempts among low-income African American adolescents. Suicide Life Threat Behav 2008;38:552-563.

29. McLaren S, Challis C. Resilience among men farmers: the protective roles of social support and sense of belonging in the depression-suicidal ideation relation. Death Stud. 2009;33:262-276.

30. Taliaferro LA, Rienzo BA, Miller MD, et al. High school youth and suicide risk: exploring protection afforded through physical activity and sport participation. J School Health. 2008;78:545-553.

31. Pettingell SL, Bearinger LH, Skay CL, Resnick MD, Potthoff SJ, Eichhorn J. Protecting urban American Indian young people from suicide. Am J Health Behav. 2008;32:465-476.

32. Khair OY, Al-Mudefer UI. The motives of attempted suicide and the diagnosis of psychiatric disorders of persons who attempted suicide. Arab J Psychiatry. 2005;16:161-172.

33. Posner K. Columbia-Suicide Severity Rating Scale-Risk Assessment Version. Personal communication.

34. Hawton K, van Heeringen K. Suicide. Lancet. 2009;373:1372-1381.

35. O'Carroll PW, Berman AL, Maris RW, Moscicki EK, Tanney BL, Silverman MM. Beyond the tower of Babel: a nomenclature for suicidology. Suicide Life Threat Behav. 1996;26:237-252.
36. Qureshi NA. Trihexyphenidyl (Artane) abuse among Saudi psychiatric patients. Ann Saudi Med. 1992;12:391-394.

37. Qureshi NA, Al Amri AH, Abdelgadir MH, El-Haraka EA. Trihexyphenidyl Dependence: a controlled investigation between users and misusers. Ann Saudi Med. 1997;17:185-190.

38. Lloyd-Richardson EE, Perrine N, Dierker L, Kelley M. Characteristics and functions of non-suicidal self-injury in a community sample of adolescents. Psychol Med. 2007;37:1183-1192.

39. Cheng Y, Tao M, Riley L, et al. Protective factors relating to decreased risks of adolescent suicidal behavior. Child Care Health Dev. 2009;35: 313-322.

40. Eskin M, Ertekin K, Dereboy C, Dirmirkiran F. Risk factors for and protective factors against adolescent suicidal behavior in Turkey. Crisis. 2007;28;131-139.

41. Centers for Disease Control and Prevention. Trends in the prevalence of suicide-related behaviors. National Youth Risk Behavior Survey: 1991-2007. http://www.brhpc.org/files/yrbs07_us_suicide_related_ behaviors_trend.pdf. Accessed June 1, 2012.

42. Landheim AS, Bakken K, Vaglum P. What characterizes substance abusers who commit suicide attempts? Factors related to Axis I disorders and patterns of substance use disorders. A study of treatment-seeking substance abusers in Norway. Eur Addict Res. 2006;12:102-108.

43. Oyefeso A, Brown S, Chiang Y, Clancy C. Self-injurious behavior, traumatic life events and alexithymia among treatment seeking opiate addicts: prevalence, patterns and correlates. Drug Alcohol Depend. 2008;98:227-234.

44. Chapman JF, Ford JD. Relationships between suicide risk, traumatic experiences, and substance use among juvenile detainees. Arch Suicide Res. 2008;12:50-61.

45. US Department of Health and Human Services. The Surgeon General's call to action to prevent suicide. Available at: http://137.187.25.243/ library/calltoaction/calltoaction.htm. Accessed June 10, 2012.

46. Eisenberg ME, Ackard DM, Resnick MD. Protective factors and suicide risk in adolescents with a history of sexual abuse. $J$ Pediatr. 2007:151:482-487.

47. Chioqueta AP, Stiles TC. The relationship between psychological buffers, hopelessness, and suicidal ideation: identification of protective factors. Crisis. 2007;28:67-73.

48. McLaren S, Challis C. Resilience among men farmers: the protective roles of social support and sense of belonging in the depression-suicidal ideation relation. Death Stud. 2009;33:262-276.

49. Pettingell SL, Bearinger LH, Skay CL, Resnick MD, Potthoff SJ, Eichhorn J. Protecting urban American Indian young people from suicide. Am J Health Behav. 2008;32:465-476.

50. Moscicki EK. Identification of suicide risk factors using epidemiologic studies. Psychiatr Clin North Am. 1997;20:499-517.

51. Duberstein PR, Conwell Y, Seidlitz L, Denning DG, Cox C, Caine ED Personality traits and suicidal behavior and ideation in depressed inpatients 50 years of age and older. J Gerontology B Psychol Sci Soc Sci. 2000;55:18-26.

52. Linehan MM. Suicidal people: one population or two? Ann NY Acad Sci. $1986 ; 487 ; 16-330$

Substance Abuse and Rehabilitation

\section{Publish your work in this journal}

Substance Abuse and Rehabilitation is an international, peer-reviewed, open access journal publishing original research, case reports, editorials, reviews and commentaries on all areas of addiction and substance abuse and options for treatment and rehabilitation. The manuscript management system is completely online and includes a very quick and fair 